the potential effectiveness of gold and iron oxide $\mathrm{NMH}$ for HCC treatment.

Methods A systematic literature search in Scopus, Pubmed, and Google Scholar were conducted to identify relevant studies in gold and iron oxide NMH for HCC cell lines published from 2008 - January 2019. A total of eight studies were included, wherein four studies used gold $\mathrm{NMH}$, and the remaining four used iron oxide $\mathrm{NMH}$. The outcome of interest is cell viability after irradiation in the presence of the nanoparticles. A pooled risk ratio with $95 \%$ confidence interval (CI) was calculated using a randomeffects model.

Results Both gold (RR 0.63; 95\% CI [0.53, 0.75]; p < 0.01; $\left.\mathrm{I}^{2}=95 \%\right)$ and iron oxide (RR $0.32 ; 95 \%$ CI [0.22, 0.48$]$; $\left.\mathrm{p}<0.01 ; \mathrm{I}^{2}=97 \%\right)$ nanoparticles exhibited effectiveness in decreasing HCC cell viability. High heterogeneity is attributed to the different classes of nanoparticles, concentration, cell lines, and irradiation duration used in the pooled studies.

Conclusions NMH using gold and iron oxide nanoparticles is effective in decreasing HCC cell viability. This meta-analysis unravels the promising potential of $\mathrm{NMH}$ as a new treatment modality against HCC. The results presented provide encouraging insights for the continuous development of $\mathrm{NMH}$ for cancer treatment. Considering that iron oxide is more costeffective than gold nanoparticles, focusing on the development of iron oxide nanoparticles for this nanomedical technology may accelerate the development and clinical application of NMH.

\section{IDDF2019-ABS-0072 APATINIB POTENTIATES IRRADIATION EFFECT VIA SUPPRESSING PI3K/AKT SIGNALING PATHWAY IN HEPATOCELLULAR CARCINOMA}

${ }^{1}$ Huilin Jin*, 'Junbin Liao, ${ }^{2}$ Lixia Xu, ${ }^{1}$ Qi Zhou, ${ }^{3}$ Sui Peng. ${ }^{1}$ Department of Liver Surgery, The First Affiliated Hospital of Sun Yat-sen University, China; ${ }^{2}$ Department of Gastroenterology and Hepatology, The First Affiliated Hospital of Sun Yat-sen University, China; ${ }^{3}$ Clinical Trials Unit, The First Affiliated Hospital of Sun Yat-sen University, China

\subsection{6/gutjnl-2019-IDDFabstracts.86}

Background Both apatinib and radiotherapy showed a valid but limited therapeutic effect in hepatocellular carcinoma (HCC). This study aimed to investigate the potential clinical utility of apatinibas a radiosensitizer and its mechanism in HCC.

Methods Four human HCC cell lines including SMMC-7721, MHCC-97H, HCCLM3 and Hep-3B were treated with apatinib, irradiation or apatinib plus irradiation. For in vitro study, colony formation assay, flow cytometry of cell cycle and apoptosis, immunofluorescence-based detection of nuclear $\gamma-\mathrm{H} 2 \mathrm{AX}$ foci, immunohistochemistry, TUNEL assay, western blot and RNA sequencing were performed. For in vivo experiment, xenograft models generated from nude mice bearing subcutaneous HCC tumors were used to assess the impact on tumor growth of apatinib plus irradiation compared with monotherapies.

Results Colony formation assay revealed that apatinib enhanced the radiosensitivity of HCC cell lines with sensitivity enhancement ratio (SER) in SMMC-7721, MHCC-97H, HCCLM3 and Hep-3B, respectively. Immunofluorescence and western blot displayed that nuclear $\gamma$-H2AX foci exhibited more and lasted longer in the combination treatment group than that in monotherapies groups, indicating apatinib suppressed repair of radiation-induced DNA double-strand breaks. Flow cytometry analysis showed that apatinib increased radiation-induced apoptosis. RNA sequencing and western blot detection revealed that apatinib radiosensitized HCC cells via suppression of radiation inducing PI3K/AKT pathway, which was further confirmed by using PI3K or AKT inhibitors. Moreover, in vivo study indicated xenograft tumor growth was significantly decreased in the combination treatment group compared with mono apatinib or irradiation group.

Conclusions Our results demonstrate that apatinib could sensitize HCC radiotherapy through inhibiting PI3K/AKT signaling pathway. Apatinib may have potential as a radiosensitizer to augment the clinical therapeutic effect of radiotherapy in HCC.

\section{IDDF2019-ABS-0074 PHYTOFABRICATED SILVER NANOPARTICLES OF PHYLLANTHUS EMBLICA EXTRACT ATTENUATED DIETHYLNITROSAMINE INDUCED HEPATOCELLULAR CARCINOMA IN RATS VIA NF-KB PATHWAY}

${ }^{1}$ Deepika Singh*, ${ }^{2}$ Manvendra Singh, ${ }^{3}$ Niti Singh. ${ }^{1}$, Sam Higginbottom University of Agriculture, Technology and Sciences, Allahabad, India; ${ }^{2}$ HMFA-MIET, Abdul Kalam Technical University, Lucknow, India; ${ }^{3}$ Continental Pvt. Ltd, Bangalore, India

\subsection{6/gutjnl-2019-IDDFabstracts.87}

Background Hepatic cancer is one of the deadly diseases in which multiplication of cell takes place in the liver and major cause of cancer-related mortality. The important function of $\mathrm{NF}-\kappa \mathrm{B}$ is to regulate inflammatory responses by regulating activation, survival and proliferation of the cell. Subsequently, natural based products play an important role in suppressing the proinflammatory mediators by nuclear factor-kappa B (NF$\kappa \mathrm{B})$ pathway. Phyllanthus emblica used in the therapy of hepatocellular carcinoma. So, the current study was designed to scrutinize the protective effects of biofabricated silver nanoparticles (PEAgNPs) using Phyllanthus emblica aqueous leaves extract as NF-kB inhibitors for hepatic cancer.

Methods Fabricated silver nanoparticles using Phyllanthus Emblica aqueous extract (PEAgNPs) acts as reducing and capping agent. Biofabricated silver nanoparticles (AgNPs) were characterized by UV spectroscopy, X-ray diffraction, and Field Emission Scanning Electron Microscope with Energy Dispersive X-ray Analysis. Hepatic cancer was induced by diethylnitrosamine (a carcinogen) and further treated with PEAgNPs for 16 weeks. The anticancer effects were assessed via in vitro cytotoxic activity on HUH (hepatic cancer) cell line and by measuring a biochemical parameter in the Wistar rats.

Results In this study, PEAgNPs showed a peak at $418 \mathrm{~nm}$ in UV spectrophotometer with a spherical structure and gives a crystalline peak of silver in XRD pattern. Biosynthesized AgNPs significantly down-regulated the serum marker enzymes, elevated the levels of the enzymatic and non-enzymatic antioxidant profile, and diminish the levels of inflammatory markers (IL-6, TNF- $\alpha$ and IL-1 $\beta$ ) via NF- $\kappa$ pathway.

Conclusions Biofabricated silver nanoparticles exhibited remarkable productive impacts against hepatic cancer in rats and serve as a novel targeted therapy for hepatic cancer by regulating cytokines and NF- $\mathrm{KB}$. 